\title{
Barrett's: Does radiofrequency ablation reduce the need for a follow-up?
}

\section{(ㄷ)(요 $\odot$}

\author{
Authors \\ Jan Martinek ${ }^{1,2,3}$, Jana Krajciova ${ }^{1,2}$ \\ Institutions \\ 1 Department of Hepatogastroenterology, Institute for \\ Clinical and Experimental Medicine, Prague, Czech \\ Republic \\ 2 Charles University, Institute of Physiology, First Faculty \\ of Medicine, Prague, Czech Republic \\ 3 Faculty of Medicine, University of Ostrava, Ostrava, \\ Czech Republic
}

\author{
Bibliography \\ DOI https://doi.org/10.1055/a-0996-8336 | \\ Endoscopy International Open 2019; 07: E1483-E1486 \\ (c) Georg Thieme Verlag KG Stuttgart · New York \\ eISSN 2196-9736 \\ Corresponding author \\ Assoc. Prof. Jan Martinek, MD, PhD, AGAF, Department of \\ Hepatogastroenterology, Institute for Clinical and \\ Experimental Medicine, Videnska 1958/9, 14021 Prague 4 \\ Fax: +00420261364016 \\ jan.martinek@volny.cz
}

Barrett's esophagus (BE) is a well-known premalignant condition.

The diagnosis of BE is made if the distal esophagus is lined with columnar epithelium with a minimum length of $1 \mathrm{~cm}$ (tongues or circular) containing specialized intestinal metaplasia (IM) at histopathological examination [1]. BE is associated with a 30to 50 -fold increased risk of developing esophageal adenocarcinoma, which has an annual incidence of $0.12 \%$ to $0.2 \%$ in patients without intraepithelial neoplasia (IEN) [2,3]. For this reason, endoscopic surveillance with biopsies of metaplastic epithelium has been recommended to detect dysplasia or cancer at an early stage, when it is still curable, preferably endoscopically.

At present, the approach to a patient with BE depends mainly on presence of IEN, which increases risk of development of esophageal adenocarcinoma (patients with confirmed lowgrade IEN have a risk of progression to high-grade IEN or adenocarcinoma $13.4 \%$ per year and in patients with high-grade IEN, risk of developing adenocarcinoma increases to approximately $10 \%$ to $20 \%$ per year).

Patients without IEN should be provided with antireflux therapy and undergo endoscopic surveillance. European Society of Gastrointestinal Endoscopy guidelines from 2017 recommend varying surveillance intervals for different $B E$ lengths. For $B E$ $\geq 1 \mathrm{~cm}$ and $<3 \mathrm{~cm}$, BE surveillance should be repeated every 5 years. For $B E \geq 3 \mathrm{~cm}$ and $<10 \mathrm{~cm}$, the interval for endoscopic surveillance should be 3 years. Patients with BE with a maximum extent $\geq 10 \mathrm{~cm}$ should be referred to a BE expert center for surveillance endoscopies [1]. It is important to note that before entering these intervals, a patient should undergo two high-quality endoscopies performed 6 months apart to reliably exclude presence of IEN. In our center, we usually follow patients with "short" segment BE every 3 to 5 years (individual adjustment based on other risk factors such as obesity, family history, presence/absence of esophagitis etc.) and patients with “long" segment BE every 2 to 3 years.

If IEN (or early cancer) is detected and confirmed by a specialized "esophageal" pathologist, patients are candidates for endoscopic treatment - endoscopic resection (ER) or endoscopic submucosal dissection (ESD) of all visible lesions and/or ablation therapy for flat Barrett's mucosa. ER/ESD is indicated in all patients with histologically proven cancer and RFA is indicated after ER/ESD to ablate residual Barrett's mucosa.

Thus, radiofrequency ablation (RFA) is the first-line treatment for patients with flat BE with confirmed low- or highgrade IEN and for patients after curative ER/ESD of early adenocarcinoma (EAC) or any other visible abnormalities to eradicate the remnant metaplastic epithelium and prevent recurrence of neoplasia and/or of BE [1,3].

RFA uses thermal energy generated by a radiofrequency current to destroy the diseased tissue and is currently the most effective and standard method of ablation used in patients with BE-related neoplasia. Aims of RFA (and of other endoscopic treatment in general) are to achieve complete eradication and remission of both IEN and IM, meaning complete disappearance of BE macroscopically and also microscopically. 


\section{What are the limitations of RFA?}

As in the case of other treatments in medicine in general, RFA has some shortcomings and limitations:

1. RFA is not free from adverse events, the most frequent being strictures, which may sometimes be refractory.

2. The effectiveness of RFA is not $100 \%$; some patients have persistent neoplasia or metaplasia.

3. RFA is not a one-step treatment and multiple sessions may be required, with some patients needing even up to five treatment sessions. This may be demanding in terms of patient compliance and cost.

4. Recurrences of both BE and neoplasia may occur, therefore, patients with successful treatment (i. e. disappearance of BE) should undergo further surveillance even if recurrences are rather infrequent.

Orman et al. [4] showed in a meta-analysis of 18 studies with 3802 patients that complete remission (CR) of IM and IEN was achieved in $78 \%(95 \% \mathrm{Cl} 70-86)$ and $91 \%$ (95\% Cl $87-95)$, respectively. IM recurrence occurred in $13 \%$ (95\% Cl 9-18). Neoplasia recurred in nine patients (4 patients had cancer) with an overall risk of $0.9 \%$ (IEN) and $0.7 \%$ (cancer), respectively, over 1.5 years.

In our recently published analysis from the Czech National Database we found that among patients with BE-related neoplasia (BORN) who had undergone endoscopic treatment consisting of RFA with or without ER (or ESD), $98.5 \%$ patients achieved complete remission of neoplasia (CR-N) and $77.9 \%$ patients achieved complete remission from intestinal metaplasia (CR-IM) [5]. Neoplasia recurred in six patients $(4.5 \%, 6 / 134)$ and intestinal metaplasia recurred in 16 patients $(15 \%, 16 / 106)$ ( $\vee$ Fig. 1). A total of seven patients had macroscopic recurrence of BE. The majority of patients with persistent or recurrent IM did not have macroscopically visible BE, thus, persistent/recurrent IM within a normal neo-Z-line does not probably represent a risk factor for neoplastic progression (but we are not $100 \%$ sure).

In our study, all patients with recurrent neoplasia (low or high-grade IEN, no patients had cancer recurrence) were successfully treated endoscopically (ER or re-RFA), it is obvious that without surveillance endoscopies, these recurrences would not have been detected and these patients could have had disease progression to cancer.

Similar results have been reported from several studies, with IM recurrence rate at $2 \%$ to $20 \%$ and with neoplasia recurrence rate at $2 \%$ to $7 \%$. Post-RFA cancer is diagnosed rarely. For example, the UK RFA registry reported overall post-treatment progression to cancer in 2\% (4/198 patients).

In summary, post-RFA (or post-endoscopic treatment in general) recurrences of neoplasia/BE occur and therefore, all patients after successful treatment still need endoscopic surveillance. The exceptions should be assessed individually; very old patients or patients with severe comorbidity do not have to be further surveilled.

Unfortunately, risk factors for recurrent IM/neoplasia/BE have not been defined yet. There is some evidence that overall recurrence rates are higher in patients with advanced histopathological diagnosis (e.g. cancer vs. low-grade IEN or no IEN). In our study, according to a multivariate logistic regression analysis adjusted for age, gender and BE length, the diagnosis of cancer was an independent risk factor for recurrent IM after RFA (OR 7.0, $95 \% \mathrm{Cl} 1.6-30.9, P<0.0005$ ) [5].

It is likely that ongoing reflux is also the risk factor for recurrence and, therefore, after successful treatment of BORN, all patients need an effective antireflux treatment [1].

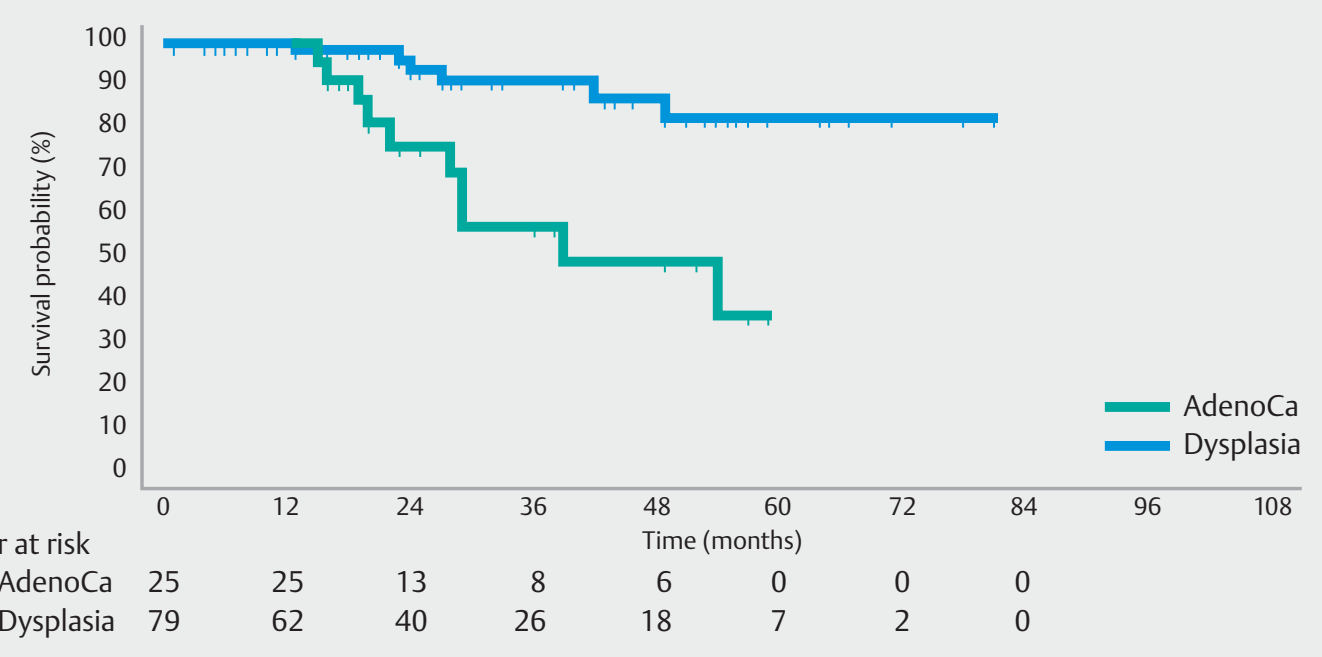

Fig. 1 Kaplan-Meier analysis of durability of complete remission of intestinal metaplasia according to the primary diagnosis [5]. 


\section{How to follow patients after successful RFA?}

The principal question remains what should be the interval of endoscopic surveillance after endoscopic therapy of BORN.

The surveillance intervals should be based on the risk of recurrent BE/neoplasia.The proposed surveillance intervals vary between different guidelines and have been based on expert opinions. Earlier recommendation seemed "relatively aggressive" based on the real risk of recurrence. For example, in 2016, the American College of Gastroenterology recommended that for patients with high-grade IEN or adenocarcinoma, endoscopies should be performed every 3 months for the first year (after successful treatment), every 6 months in the second year, and annually thereafter. For patients with an initial diagnosis of low-grade IEN, endoscopic surveillance was recommended every 6 months in the first year and annually thereafter [6].

Cotton et al. tried to define new intervals for surveillance endoscopy in patients after successful treatment of BORN based on data from both United States and UK registries [7]. They found that recurrent neoplasia is best predicted by the patient's worst histologic grade prior to entering treatment and surveillance. Based on risk of recurrences, they proposed a much-attenuated schedule of surveillance post-RFA endoscopy, which should provide sufficient protection from adenocarcinoma with less harm and cost due to "oversurveillance." In their study, the annual rate of recurrent neoplasia was $1.98 \%$ in patients with an initial diagnosis of low-grade IEN and $5.93 \%$ in patient with high-grade IEN or cancer ( $>$ Fig. 2 ).

They proposed the following intervals for surveillance endoscopy:

- Patients with low-grade IEN: at 1 and 3 years after CR-IM and then after 5 years.
- Patients with high-grade IEN or EAC: at 3 months, 6 months and 1 year after CR-IM and then annually (up to 7 years).

In our center, we follow patients according to baseline histopathology:

- EAC every 3 to 6 months during the first 2 years, then annually for three years and then every 2 to 3 years.

- High-grade IEN every 6 months during the first year and then annually for 3 years, then every 2 to 3 years.

- Low-grade IEN: at 1 and 2 years, then every 3 to 5 years.

If we compare those intervals with standard surveillance intervals of patients with BE without IEN, there is no major reduction of surveillance endoscopies, in contrast, there is an increase in endoscopies needed. But most important and relevant is that, in our view, is the number of successfully treated patients who did not have to undergo a riskier surgery and therefore run the risk of mortality owing to esophageal cancer. Post-RFA surveillance contributes to this great achievement in gastroenterology.

\section{Conclusion}

Results with RFA are good, but we must accept the fact that there are a few cases with BORN recurrence. Thus after successful RFA, patients still need endoscopic surveillance. Intervals of surveillance endoscopies are still a matter for debate and should depend principally on pretreatment histology. The most frequent endoscopies should be performed in patients with early carcinoma (higher risk for recurrence) followed by patients with high-grade IEN and the least frequent being in patients with initial low-grade IEN (patients without IEN, who undergo RFA, do not probably need a strict endoscopic surveillance). The main reason for continuing endoscopic follow-up is early detection of recurrence, when it is treatable endoscopically.

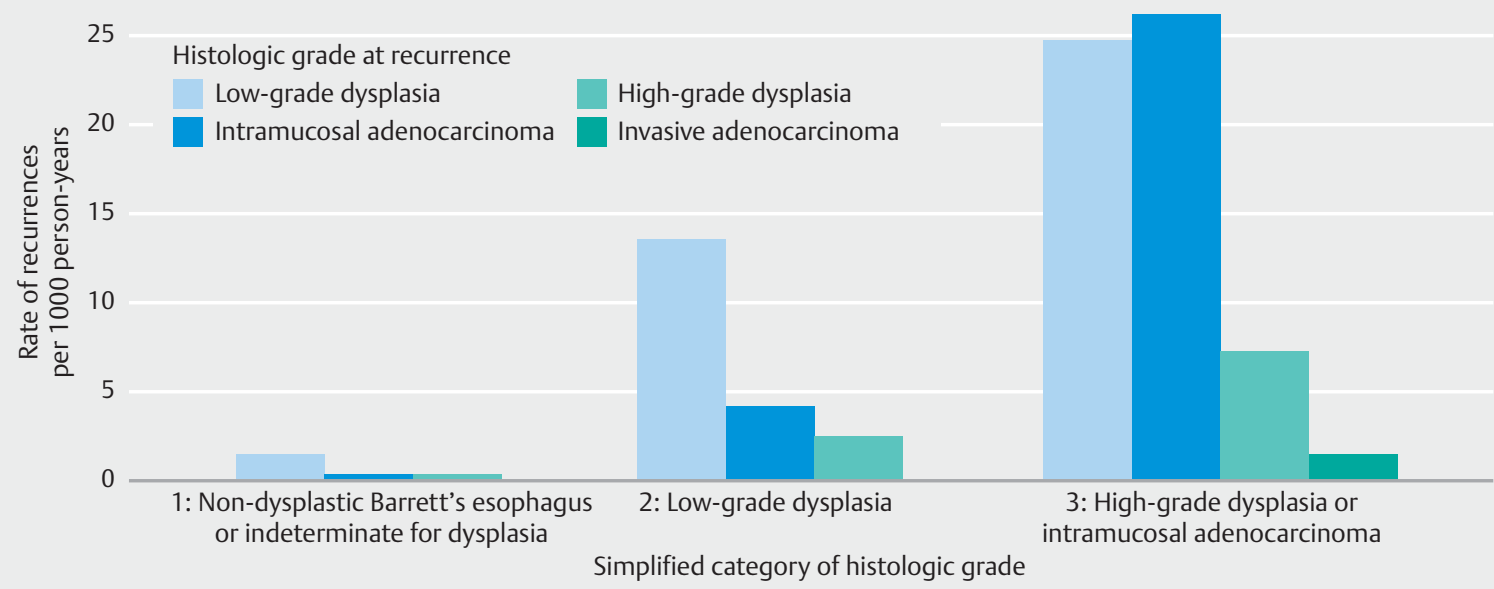

- Fig. 2 Risk of recurrences of intraepithelial neoplasia/cancer based on initial histopathological diagnosis. Risk of recurrence significantly increases from no-IEN to high-grade IEN and cancer [7]. 


\section{Competing interests}

None

\section{References}

[1] Weusten B, Bisschops R, Coron E et al. Endoscopic management of Barrett's esophagus: European Society of Gastrointestinal Endoscopy (ESGE) Position Statement. Endoscopy 2017; 49: 191 - 198

[2] Martinek J, Benes M, Brandtl P et al. Low incidence of adenocarcinoma and high-grade intraepithelial neoplasia in patients with Barrett's esophagus: a prospective cohort study. Endoscopy 2008; 40: 711 716
[3] Shaheen N], Sharma P, Overholt BF et al. Radiofrequency ablation in barrett's esophagus with dysplasia. N Engl J Med 2009; 360: 2277 2288

[4] Orman ES, Nan LI, Shaheen NJ. Efficacy and durability of radiofrequency ablation for barrett's esophagus: systematic review and meta-analysis. Clin Gastroenterol Hepatol 2013; 11: 1245-1255

[5] Krajciova J, Janicko M, Falt P et al. Radiofrequency ablation in patients with Barrett's esophagus-related neoplasia - long-term outcomes in the Czech National Database. J Gastrointest Liver Dis 2019; 28: 149155

[6] Shaheen NJ, Falk GW, lyer PG et al. ACG Clinical Guideline: Diagnosis and Management of Barrett's esophagus. Am J Gastroenterol 2016; 11: $30-50$

[7] Cotton CC, Haidry R, Thrift AP et al. Development of evidence-based surveillance intervals after radiofrequency ablation of Barrett's esophagus. Gastroenterology 2018; 155: 316 -326.e6 